MODELING, IDENTIFICATION AND CONTROL, 1982, vOL. 3, NO. 4, 211-222

doi:10.4173/mic.19824.2

\title{
Optimal evasive manoeuvre for a ship in an environment of fixed installations and other ships
}

\author{
ROLF SKJONG† and KÅRE M. MJELDE $\dagger$ \\ Keywords: optimal control, differential game, offshore, ship, collision, manoeuvre. \\ Collision avoidance for encounters between several ships and installations in the \\ open sea, is treated as a problem of optimal control, using the theory of differential \\ games. Each ship is, in this idealized model, assumed to have two controls \\ corresponding to rudder angle and engine setting. The objective function, which \\ the shipmasters try to minimize in an optimal evasive manoeuvre, is defined as the \\ collision risk. Numerical solutions for the $M$-ships and $I$-installations optimal \\ evasive manoeuvre problem, can be found by the ping-pong algorithm. Numerical \\ examples are given for up to five ships and two installations.
}

\section{Introduction}

This paper is a pilot study of a broader project on collision risk between ships and offshore installations. Our main interest has been to determine how the frequency of collisions, and hence the safety and economy of ship operations, depends on the manoeuvring capabilities of the ships involved, the complexity of the installation geometry and the complexity of the traffic situation. To this end it has been found necessary to develop a mathematical model of collision avoidance and occurrence. A reformulation of the model has also been suggested for ship-based collision avoidance systems.

The mathematical model presented in this paper is rather general concerning the number of ships involved in the encounter situation. Problems with many ships can be treated. The frequency of three ships' encounters, where rules of the road at sea are undefined, increases as the cube of the ship density. This important relation was first noticed by Jensen (1970) in an investigation concerning accidents in the channel between Denmark and Sweden. This relation was also discussed by Stratton (1971) in a study on traffic in UK waters.

As did Miloh and Sharma (1975), we employ the theory of Differential Games as formulated by Isaacs (1965) as our theoretical foundation. Although this theory was originally developed for solving military problems of guided pursuit and dogfighting, the same formulation can be applied to problems of collision avoidance. The main difference between the military application and the collision avoidance application is that in problems of guided pursuit the pursuer tries to hit and the evader tries to escape, while in the case of collision avoidance all the shipmasters should try to evade in order to minimize the collision risk.

\section{Differential game formulation}

\subsection{Basic Concepts}

The theatre of operation is a region in the Euclidian $n$-space. We think of a particular point $x=\left\{x_{1} \ldots x_{n}\right\}$ to be in motion in state space, its path being governed 
by the kinematic equation. The state variables specify the current state of the system. The kinematic equations relate the time rate of change of state variables to the state variables themselves and to control variables, which are at the volition of the players.

The game is characterized by a payoff. A payoff comprises a terminal component as a function of the terminal conditions and/or an integral component as a function of the path along which the terminal conditions are reached. The objective of an individual player is to maximize (or minimize) the payoff. It is assumed that each player has full information on the current state of the system, and that the game is non-negotiable, i.e. no coalition is allowed.

A function relating the control-variables to the state variables is called a strategy. If all players play their mutually optimal strategies, there exists for each initial condition a conceptually predetermined unique payoff which Isaacs called the Value (Capital ' $V$ '). The theoretical solution of the game comprises the optimal strategies, the optimal paths and the Value as a function of initial conditions. A continuous payoff function yields a game of degree with a continuous value function. A discrete value function yields a game of kind with the state space subdivided into zones of different Values separated by barriers.

While previous work along these lines have used terminal payoff (Miloh 1974, Miloh and Sharma 1975, Vincent et al. 1972, and Merz 1973) and a game of kind, we use an integral payoff in a game of degree. The benefits of this will appear throughout this study since our formulation can be solved without painstaking analytical work in the more complicated situations.

\subsection{Kinematic equations}

In our preliminary and highly idealized mathematical model, our dynamic system consists of $M$-ships manoeuvring on a homogeneous, isotropic, unbounded and undisturbed sea surface with $I$-installations against which collisions should be avoided. We may add, however, that incorporation of wind and wave forces scarcely influences the complexity of the formulation and the solution algorithm.

For each ship we choose to describe the motion in the 4- $M$-dimensional space by the following set of kinematic equations:

$$
\begin{aligned}
& \dot{x}_{i}=w_{i} \cos \theta_{i} \\
& \dot{y}_{i}=w_{i} \sin \theta_{i} \\
& \dot{\theta}=\phi_{i} w_{i} / R_{i} \\
& \dot{w}_{i}=\psi_{i} T_{i} / m_{j}-\left|w_{i}\right| w_{i} k_{i} / m_{i} \\
& \forall i \in M
\end{aligned}
$$

where

$$
\dot{x}=\frac{\mathrm{d} x}{\mathrm{~d} t} \quad \text { etc. }
$$

The state variables are the rectilinear coordinates, the course angle and the absolute forward speed $(x, y, \theta, w)$. 
The four fixed parameters for each ship are the minimum turning radius $R_{i}$, the maximum propulsive thrust $T_{i}$, the effective mass $m_{i}$ and the coefficient of resistance $k_{i}$. The two control variables which are at the shipmasters' disposal are $\Phi_{i}$, a normalized radius of path-curvature and $\psi_{i}$, a normalized propulsive thrust. We have $\Phi_{i} \in[-1,1]$ and $\psi_{i} \in[-1,1]$. From eqn. (4) the stopping time $T_{i}$ and the stopping distance $D_{i}$ can be evaluated.

$$
\begin{aligned}
& T_{i}=\frac{m_{i}}{\sqrt{ }\left(T_{i} k_{i}\right)} \operatorname{arctg}\left(\sqrt{ }\left(k_{i} / T_{i}\right) \bar{w}_{i}\right)<\frac{\pi}{2} \frac{m_{i}}{\sqrt{ }\left(T_{i} k_{i}\right)} \\
& D_{i}=\int_{0}^{T} w(t) \mathrm{d} t=\frac{1}{2} \frac{m_{i}}{k_{i}} \ln \left(1+\frac{k_{i}}{T_{i}} \bar{w}_{i}^{2}\right)
\end{aligned}
$$

where $\bar{w}_{i}$ is the initial velocity.

\subsection{Payoff}

To complete the formulation of the differential game we must now specify the objective of our players. The best case of evasion is that all players try to minimize the total collision risk. We define the increase in collision risk $(\mathrm{d} P)$ between the players or objects $i$ and $j$ during the time $\mathrm{d} t$ as:

$$
\mathrm{d} P_{i j}=k_{i j}\left|\hat{w}_{i j}\right| \mathrm{d} t \iint_{R^{2}} \mathrm{~d} x \mathrm{~d} y \rho_{t}(x, y, t) \rho_{j}(x, y, t)
$$

where $k_{i j}$ is a normalization constant of dimension length, and $\hat{w}_{i j}$ is the radial velocity for the two object $i$ and $j$, i.e.:

$$
\hat{w}_{i j}=\frac{\mathrm{d}}{\mathrm{d} t} r_{i j}(t)
$$

where

$$
r_{i j}(t)=\mathcal{V}^{\prime}\left(\left(y_{j}(t)-y_{i}(t)^{2}+\left(x_{j}(t)-x_{i}(t)\right)^{2}\right)\right.
$$

It is easily realized that

$$
\left|\hat{w}_{i j}\right|=\frac{\left|\left(x_{j}-x_{i}\right)\left(w_{j} \cos \theta_{j}-w_{i} \cos \theta_{i}\right)+\left(y_{j}-y_{i}\right)\left(w_{j} \sin \theta_{j}-w_{i} \sin \theta_{i}\right)\right|}{r_{i j}}
$$

Furthermore, $\rho_{i}$ and $\rho_{j}$ are the probability density functions for the two objects normalized as:

$$
\iint_{R^{2}} \mathrm{~d} x \mathrm{~d} y \rho_{i}(x, y, t)=1
$$

The use of a distribution for each ship can be justified from the observation that the ships' positions are never exactly known. Our main motivation for introducing this description is, however, the more obvious one of the avoidance of a discontinuity in the problem introduced by other authors. Miloh and Sharma (1975), for instance, use circular discs to represent ships. A representation similar to ours has previously been 
employed profitably by Mjelde (1977) to represent a single ship by a Poisson process with expected value 1, and by Schroeter (1976) to represent one single target in a problem of the analysis of aiming and firing strategies.

We use the normal distribution for $\rho_{l}$, i.e.

$$
\rho_{t}(x, y, t)=\frac{1}{2 \pi l_{i}^{2}} \exp \left[-\frac{1}{2} \frac{\left(x-x_{t}(t)\right)^{2}+\left(y-y_{i}(t)\right)^{2}}{l_{i}^{2}}\right]
$$

where $l_{i}$ is a typical radius of the object and $\left(x_{i}(t), y_{i}(t)\right)$ are the coordinates at time $t$ for the object. The overlap in equation (7) can be integrated out:

$$
\begin{gathered}
\iint_{R^{2}} \mathrm{~d} x \mathrm{~d} y \rho_{t}(x, y, t) \rho_{j}(x, y, t) \\
=\frac{1}{2 \pi l_{i}{ }^{2}} \frac{1}{2 \pi l_{j}^{2}} \iint_{R^{2}} \mathrm{~d} x \mathrm{~d} y \exp \left[-\frac{1}{2} \frac{\left(x-x_{i}(t)\right)^{2}+\left(y-y_{i}(t)\right)^{2}}{l_{i}^{2}}+\frac{\left(x-x_{j}(t)\right)^{2}+\left(y-y_{j}(t)\right)^{2}}{l_{j}^{2}}\right] \\
=\frac{1}{2 \pi\left(l_{i}{ }^{2}+l_{j}^{2}\right)} \exp \left[-\frac{1}{2} \frac{r_{i j}{ }^{2}(t)}{l_{i}^{2}+l_{j}^{2}}\right]
\end{gathered}
$$

The collision risk is then determinated as an integral from an initial time $\left(t_{i}\right)$, where the objects are at some distance (a few typical lengths or more), to a final time $t_{f}$. The timelapse $\left(t_{f}-t_{i}\right)$ should be long enough for the collision risk to be over at time $t_{f}$. We have:

$$
P_{i j}\left(t_{f}\right)=P_{i j}\left(t_{i}\right)+\frac{k_{i j}}{2 \pi\left(l_{i}^{2}+l_{j}^{2}\right)} \int_{t i}^{t_{f}} \mathrm{~d} t\left|\hat{w}_{i j}\right| \exp \left[-\frac{1}{2} \frac{r_{i j}^{2}(t)}{l_{i}^{2}+l_{j}^{2}}\right]
$$

We renormalize the last expression to give a collision risk of 'one' corresponding to the objects colliding head on, i.e.

$$
\frac{k_{i j}}{2 \pi\left(l_{i}^{2}+l_{j}^{2}\right)} \int_{-\infty}^{\infty} \mathrm{d} t\left|\hat{w}_{i j}\right| \exp \left[-\frac{1}{2} \frac{r_{i j}^{2}(t)}{l_{i}^{2}+l_{j}^{2}}\right]=1
$$

With $w_{t}$ and $w_{j}$ constant during the short time interval of significant contribution to the integral, we have:

$$
\frac{k_{i j}\left|w_{i}+w_{j}\right|}{2 \pi\left(l_{i}^{2}+l_{j}^{2}\right)} \int_{-\infty}^{\infty} \exp \left[-\frac{1}{2} \frac{\left(w_{i}+w_{j}\right)^{2} t^{2}}{l_{i}^{2}+l_{j}^{2}}\right] \mathrm{d} t=1
$$

which implies that:

$$
k_{i j}=\sqrt{ }\left(2 \pi\left(l_{i}^{2}+l_{j}^{2}\right)\right)
$$

An 'overtaking' collision would also give collision risk of 'one' in this formulation, i.e. $r_{i j}{ }^{2}=\left(w_{i}-w_{j}\right)^{2} t^{2}$.

To minimize the collision risk is therefore in this context to minimize the integral

$$
P_{i j}=\frac{1}{\sqrt{\left(2 \pi\left(l_{i}^{2}+l_{j}^{2}\right)\right)}} \int_{i_{i}}^{t_{f}} \mathrm{~d} t\left|\hat{w}_{i j}\right| \exp \left[-\frac{1}{2} \frac{r_{i j}^{2}(t)}{l_{i}^{2}+l_{j}^{2}}\right]
$$


When more than two objects are involved in the collision avoidance process all the players should try to minimize the total collision risk

$$
P=\sum_{i}^{M} \sum_{j=i+1}^{M+I} P_{i j}
$$

where $M$ is the number of ships and $I$ the number of installations, buoys and other fixed objects to be avoided.

\section{Analytical solution}

Following Isaacs (1965) optimal play is governed by the equation (Isaacs, The Main Equation):

$$
\begin{aligned}
\min _{\substack{\phi_{i} \\
\forall i \in M}} \min _{\psi_{i}}\left\{\sum _ { i = 1 } ^ { M } \left(V_{x_{i}} w_{i} \cos \theta_{i}+V_{y_{i}} w_{i} \sin \theta_{i}+V_{\theta_{i}} \Phi_{i} w_{i} / R_{i}\right.\right. \\
\left.+V_{w_{i}}\left(\psi_{i} T_{i} / m_{i}-\left|w_{i}\right| w_{i} k_{i} / m_{i}\right)\right)+\sum_{i}^{M} \sum_{j=i+1}^{M+I} \frac{1}{\sqrt{ }\left(2 \pi\left(l_{i}^{2}+l_{j}^{2}\right)\right)}\left|\hat{w}_{i j}\right| \\
\left.\quad \exp \left[-\frac{1}{2} \frac{r_{i j}^{2}}{\left(l_{i}^{2}+l_{j}^{2}\right)}\right]\right\}=0
\end{aligned}
$$

where $V$ is the unknown value function (i.e. $V=P=\sum_{i j} P_{i j}$ at optimal play), and

$$
V_{x}=\frac{\mathrm{d} V}{\mathrm{~d} x}
$$

The solution of eqn. (20) comprises the solution of the game.

\subsection{A property of optimal strategies}

The optimal strategies can be found immediately. The control variables appear linearly in the main equation and therefore the controls are piecewisely constant and are, in general, equal to an extreme value -1 or +1 , with the possible exception of singular cases where the expression in the parenthesis below, vanish. We have

$$
\left.\begin{array}{l}
\bar{\Phi}_{i}=-\operatorname{sgn}\left(V_{\theta_{i}} w_{i}\right) \\
\psi_{i}=-\operatorname{sgn}\left(V_{w_{i}}\right)
\end{array}\right\}
$$

where $\bar{\Phi}_{i}$ and $\psi_{i}$ are the optimal strategies, and we have employed the signum function. The effect that the controls take their extreme possible values is called the bang-bang principle; it is a consequence of the linearly appearing control variables which again is a direct consequence of our dynamic model.

\subsection{Adjoint equations}

The main equation is a first order partial differential equation. It can be solved by integrating the characteristic equations, which consist of the kinematic equations, and the adjoint equations obtained by differentiating the main equation with respect to the 
explicitly appearing state variables. For reasons to arise later we find it advantageous to write the adjoint equations with retrogressive time derivatives, i.e.

$$
\stackrel{\mathrm{d} x}{\mathrm{x}}=\frac{\mathrm{d} \mathrm{Y}}{\mathrm{r}} \text { where } \mathrm{Y}=\mathrm{const}-t
$$

The adjoint equations can be written:

$$
\begin{aligned}
& {\stackrel{\circ}{V_{i}}}_{x_{i}}=\sum_{\substack{j=1 \\
\neq i}}^{M+I} \frac{1}{\sqrt{ }\left(2 \pi\left(l_{i}{ }^{2}+l_{j}^{2}\right)\right)}\left\{\left|\hat{w}_{i j}(t)\right|\left(x_{j}-x_{i}\right)\left(\left(l_{i}{ }^{2}+l_{j}^{2}\right)^{-1}+r_{i j}{ }^{-2}\right)\right. \\
& \left.-\eta_{i j} \frac{w_{j} \cos \theta_{j}-w_{i} \cos \theta_{i}}{r_{i j}}\right\} \exp \left[-\frac{1}{2} \frac{r_{i j}^{2}}{l_{l}^{2}+l_{j}^{2}}\right] \\
& {\stackrel{\circ}{y_{i}}}=\sum_{\substack{j=1 \\
\neq i}}^{M+I} \frac{1}{\sqrt{ }\left(2 \pi\left(l_{i}^{2}+l_{j}^{2}\right)\right)}\left\{\left|\hat{w}_{i j}(t)\right|\left(y_{j}-y_{i}\right)\left(\left(l_{i}^{2}+l_{j}^{2}\right)^{-1}+r_{i j}{ }^{-2}\right)\right. \\
& \left.-\eta_{i j} \frac{w_{j} \sin \theta_{j}-w_{i} \sin \theta_{i}}{r_{i j}}\right\} \exp \left[-\frac{1}{2} \frac{r_{i j}^{2}}{l_{i}^{2}+l_{j}^{2}}\right] \\
& {\stackrel{\circ}{\theta_{i}}}^{\circ}=-V_{x_{i}} w_{i} \sin \theta_{i}+V_{y_{i}} w_{i} \cos \theta_{i} \\
& +\sum_{\substack{j=1 \\
\neq i}}^{M+I} \eta_{i j} \frac{\left(x_{j}-x_{i}\right)\left(+w_{i} \sin \theta_{i}\right)+\left(y_{j}-y_{i}\right)\left(-w_{i} \cos \theta_{i}\right)}{\sqrt{ }\left(2 \pi\left(l_{i}^{2}+l_{j}^{2}\right)\right) r_{i j}} \\
& \times \exp \left[-\frac{1}{2} \frac{r_{i j}^{2}}{l_{i}^{2}+l_{j}^{2}}\right] \\
& {\stackrel{\circ}{w_{i}}}=V_{x_{i}} \cos \theta_{i}+V_{y_{i}} \sin \theta_{i}-\operatorname{sgn}\left(V_{\theta_{i}} w_{i}\right) V_{\theta_{i}} / R_{i}-2 V_{w_{i}}\left|w_{i}\right| k_{i} / m_{i} \\
& +\sum_{j=1}^{M+I} \eta_{i j} \frac{\left(x_{j}-x_{i}\right)\left(-\cos \theta_{i}\right)+\left(y_{j}-y_{i}\right)\left(-\sin \theta_{i}\right)}{\sqrt{ }\left(2 \pi\left(l_{i}^{2}+l_{j}^{2}\right)\right) r_{i j}} \\
& \times \exp \left[-\frac{1}{2} \frac{r_{i j}^{2}}{l_{i}^{2}+l_{j}^{2}}\right]
\end{aligned}
$$

where

$$
\eta_{i j}=\operatorname{sgn}\left(\hat{w}_{i j}\right)
$$

It can easily be given upper bounds on the initial values of the adjoint variables, which numerically implies that

$$
V_{x_{i}}\left(t_{f}\right)=V_{y_{i}}\left(t_{f}\right)=V_{\theta_{i}}\left(t_{f}\right)=V_{w_{i}}\left(t_{f}\right)=0 ; \quad \forall i \in M
$$

\section{Solution algorithm}

For our solution algorithm we employ the ping-pong algorithm, which has previously been utilized to solve the apartment-heating problem by Holt and Mukundan (1972). In this context the algorithm can be formulated as follows: 
1. Determine the timelapse $\left(t_{f}-t_{i}\right)$.

2. Initially guess every player's nominal strategy for every discrete instant of time from $t_{i}$ to $t_{f}$. Of course, the better this initial guess is, the faster convergence will be attained in the solution-search.

3. Since the controls are known, the state equations can be integrated forward in time, we use a Simpson routine except for $w_{i}$ where we use analytical expressions. Values of each state variable are stored for every discrete instant of time. Integrate in this order:
(a) $w_{i}(t) \quad \forall i \in M$
(b) $\theta_{i}(t) \quad \forall i \in M$
(c) $x_{i}(t), y_{i}(t) \quad \forall i \in M$

4. Calculate the collision probabilities and store them until the next iteration.

5. Check on convergence: If the calculated collision probability is not decreasing more than a predefined $\epsilon(0<\epsilon \ll 1)$ then terminate by jumping to 9 below.

6. Integrate the adjoint equations retrogressively, realizing that the necessary initial conditions are in fact the known adjoint final conditions. This retrogressive integration calls for the stored values of the state variables obtained by forward integration. Perform the integration in the following order:
(a) $V_{x_{i}}(t) \quad \forall i \in M$ (Simpson)
(b) $V_{y_{i}}(t) \quad \forall i \in M$ (Simpson)
(c) $V_{\theta_{i}}(t) \quad \forall i \in M$ (Simpson)
(d) $V_{w_{i}}(t) \quad \forall i \in M$ (4th order Runge-Kutta)

7. Calculate the new control variables for all times throughout the interval from $t_{i}$ to $t_{f}$, by using eqn. (21).

8. Return to 3.

9. Perform a perturbation control to check for equilibrium.

10. Call for output, comprising: The minimal collision risk, the optimal strategies and the optimal paths.

It is numerically consistent to use a Simpson algorithm together with a 4th order Runge-Kutta method since both are fourth order methods.

An improvement on making the procedure more efficient can be accomplished by utilizing the solution for the $M$-ships case of collision avoidance as an initial guess for the $(M+1)$-ships case.

\section{Numerical examples}

The large number of parameters and the number of initial conditions which define a particular game, generally prevents comprehensive computations. In addition, the objective of each player could be varied. This would define new sets of games. A game with one pursuer and one evader could be formulated. For the pursuer's strategy this would just give a change in sign in eqn. (21). This problem would closely resemble the homicidal chauffeur game formulated by Isaacs (1965) and investigated extensively by Breakwell and Merz (1969). 
The examples are chosen to illustrate the procedure's ability to find the optimal strategies and the optimal paths when the ships involved are all trying to take courses to minimize the total collision risk. The selected parameters for the five ships involved in the evasive manoeuvres can be found in Table $1(a)$. The parameters are typical for bulk carriers. The typical radii, which define the dimensions of the two installations in Fig. 1, are fifty metres.

\begin{tabular}{lrrrrrc}
\hline Ship & 1 & \multicolumn{1}{c}{2} & \multicolumn{1}{c}{3} & \multicolumn{1}{c}{4} & \multicolumn{1}{c}{5} & [units] \\
\hline Min. radius of curvature & $500 \cdot 0$ & $400 \cdot 0$ & $500 \cdot 0$ & $600 \cdot 0$ & $500 \cdot 0$ & metres \\
Typical radius & $50 \cdot 0$ & $75 \cdot 0$ & $50 \cdot 0$ & $50 \cdot 0$ & $50 \cdot 0$ & metres \\
Thrust & $5 \cdot 1$ & $4 \cdot 1$ & $5 \cdot 1$ & $4 \cdot 0$ & $5 \cdot 1$ & $\times 10^{5}$ Newton \\
Effective mass & $2 \cdot 6$ & $1 \cdot 5$ & $2 \cdot 6$ & $1 \cdot 5$ & $2 \cdot 6$ & $\times 10^{7} \mathrm{~kg}$ \\
Water resistance & $9 \cdot 8$ & $9 \cdot 0$ & $9 \cdot 8$ & $9 \cdot 0$ & $9 \cdot 8$ & $\times 10^{3} \mathrm{~kg} / \mathrm{m}$ \\
\hline
\end{tabular}

Table 1. (a). Manoeuvring capabilities for the ships.

\begin{tabular}{lrrrrrl}
\hline Ship & 1 & \multicolumn{1}{c}{2} & \multicolumn{1}{c}{3} & \multicolumn{1}{c}{4} & \multicolumn{1}{c}{5} & [units] \\
\hline Initial course & $180 \cdot 0$ & $270 \cdot 0$ & $0 \cdot 0$ & $0 \cdot 0$ & $0 \cdot 0$ & degrees \\
Initial velocity & $14 \cdot 0$ & $11 \cdot 2$ & $14 \cdot 0$ & $11 \cdot 7$ & $14 \cdot 0$ & knots \\
Initial $(x)$ & $500 \cdot 0$ & $0 \cdot 0$ & $-500 \cdot 0$ & $-250 \cdot 0$ & $-250 \cdot 0$ & metres \\
Coordinates $(y)$ & $0 \cdot 0$ & $399 \cdot 0$ & $0 \cdot 0$ & $-250 \cdot 0$ & $250 \cdot 0$ & metres \\
\hline
\end{tabular}

Table $1(b)$. Initial conditions in fig. (1).

\begin{tabular}{|c|c|c|c|c|c|c|c|}
\hline \multicolumn{2}{|c|}{$\begin{array}{r}\text { CPA } \\
\text { [metres] }\end{array}$} & \multicolumn{3}{|c|}{ Ships } & & \multicolumn{2}{|c|}{$\begin{array}{l}\text { Instal- } \\
\text { lation }\end{array}$} \\
\hline $\begin{array}{l}\text { Collision } \\
\text { risk }\end{array}$ & 1 & 2 & 3 & 4 & 5 & 6 & 7 \\
\hline 1 & & 40 & 402 & 281 & 220 & 187 & 241 \\
\hline 2 & 0.66 & & 380 & 80 & 95 & 165 & 320 \\
\hline 3 & $0.13 \times 10^{-7}$ & $26 \times 10^{-4}$ & & 267 & 354 & 207 & 83 \\
\hline 4 & $0.70 \times 10^{-3}$ & 0.38 & $0.16 \times 10^{-3}$ & & 198 & 192 & 250 \\
\hline 5 & $0.19 \times 10^{-2}$ & 0.52 & $0.28 \times 10^{-3}$ & $0.50 \times 10^{-2}$ & & 291 & 252 \\
\hline 6 & $0.80 \times 10^{-2}$ & $0.68 \times 10^{-1}$ & $0.34 \times 10^{-2}$ & $0.66 \times 10^{-2}$ & $0.40 \times 10^{-4}$ & & \\
\hline 7 & $0.64 \times 10^{-3}$ & $0.38 \times 10^{-3}$ & $0 \cdot 24$ & $0.24 \times 10^{-3}$ & $0.22 \times 10^{-3}$ & & \\
\hline
\end{tabular}

Table $1(c)$. Output data for fig. (1).

Since the solutions are plotted in realistic, nonreduced space the figures are almost self-explanatory. Vectors on the figures are representing the ships at one minute intervals with length of the vector representing velocities and their directions representing the course of the ships. In addition small vectors appended on these velocity vecors indicate the direction of the propulsive thrust. The small triangles at the paths indicate the passage of a transition surface for the thruster controls. 


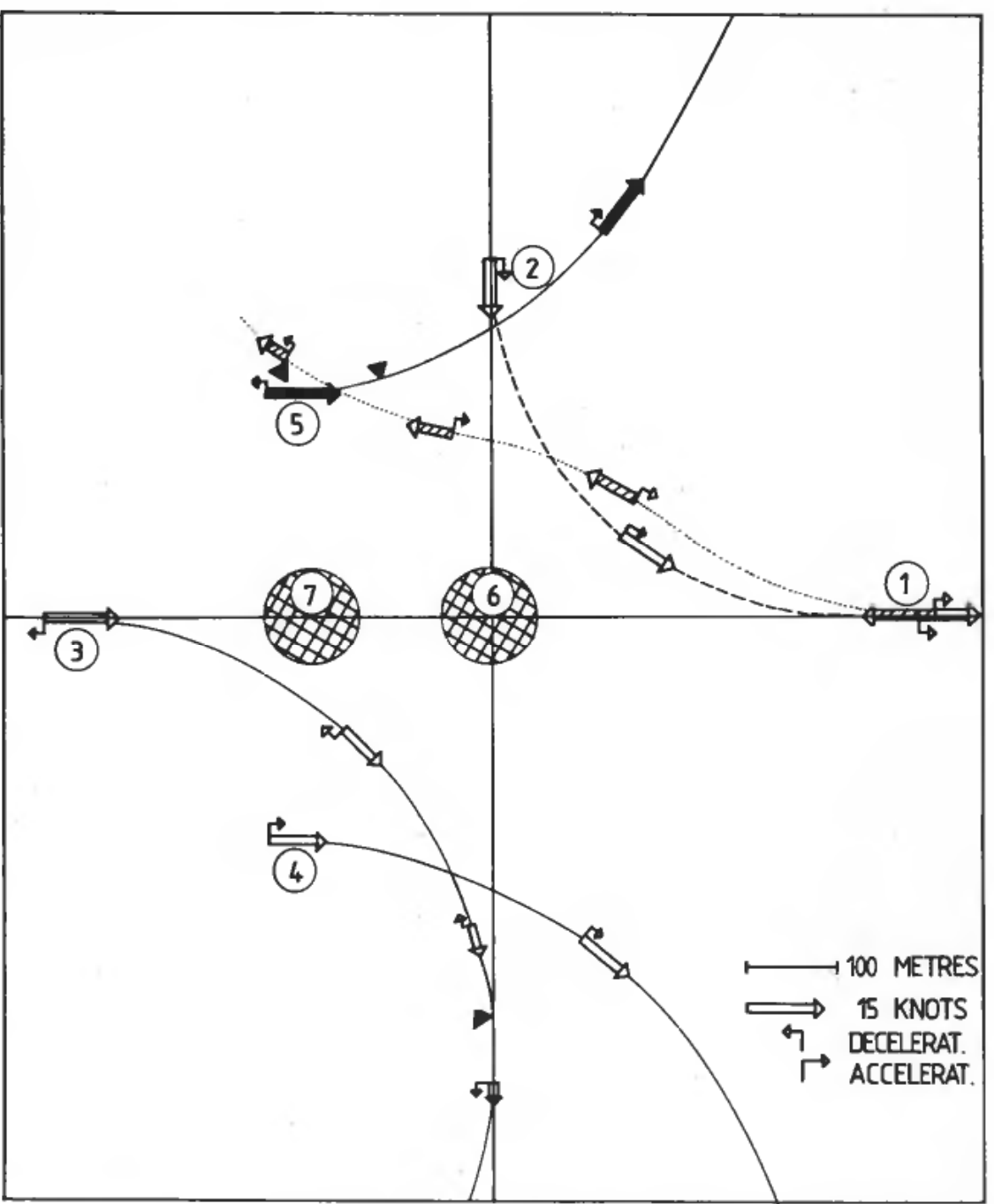

Figure 1. Five ships' and two installations' encounter. The input data can be found in Tables $1(a)$ and $1(b)$, the output data in Table $1(c)$.

From the large sample of collision avoidance scenarios which we have run on the computer, we give two illustrative examples. Figure 1 gives an example with five ships and two installations and Fig. 2 gives an example of a rather extreme situation of a five ship encounter in open sea. In Fig. 1 the two ships 1 and 2 have no better alternative than to choose strategies that give a close passage. The thruster controls are, however, used to widen the passage as much as possible, with ship 1 decelerating, and ship 2 accelerating. The reason that ship 1 turns starboard in the first place is that the other choice of rudder control would bring it close to both ship 3 and ship 4 . In both figures one can observe examples on transition surfaces for the thruster control for one ship astern the closest of the other ships. This is in good agreement with intuition; the ship decelerates to let the other ship pass and then starts to accelerate.

The extreme situation in Fig. 2 cannot be successfully resolved even when the shipmasters act optimally. The two ships 3 and 5 would probably collide even at 


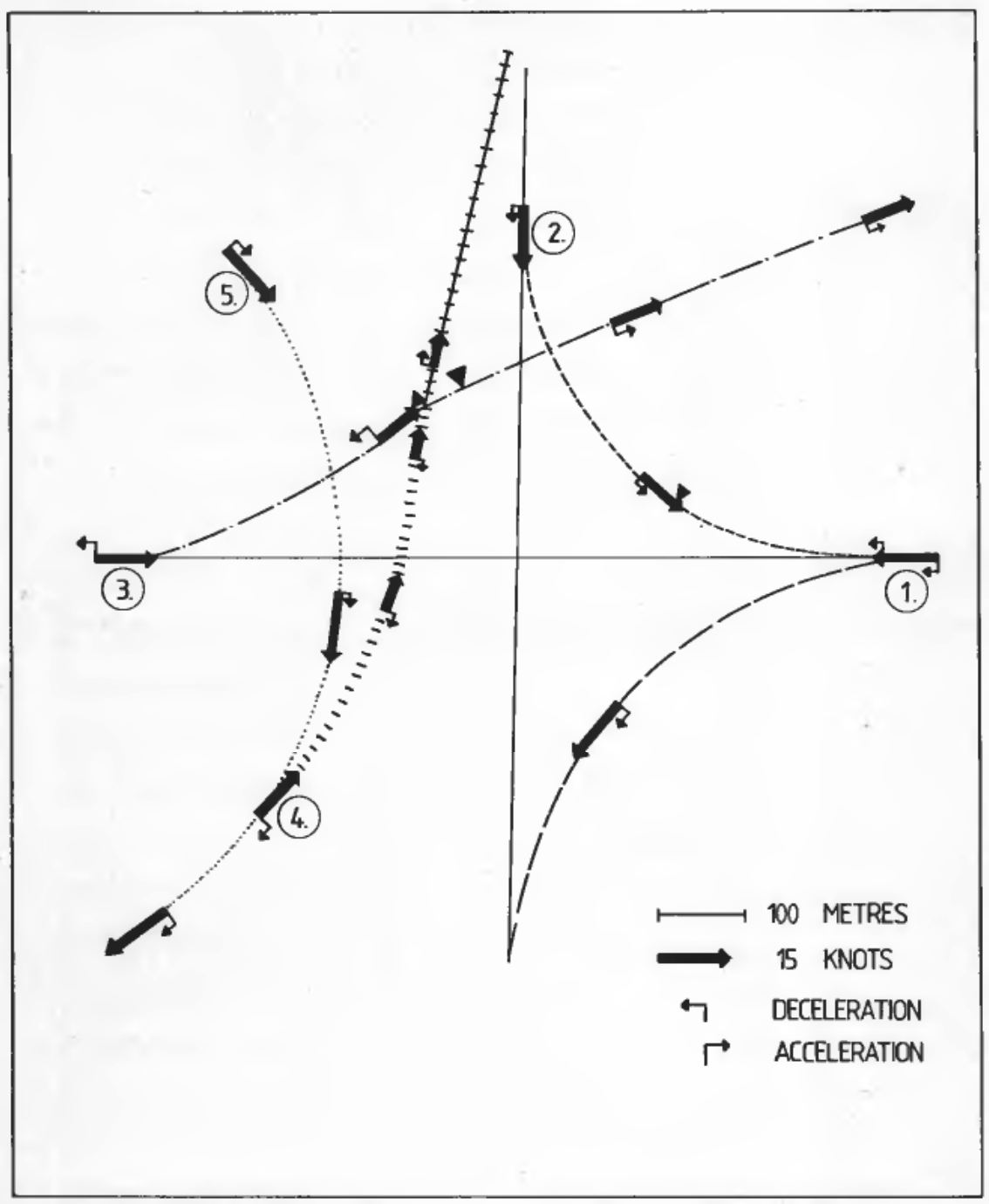

Figure 2. Five ships' encounter in open sea. Initially all five ships are on courses towards collision. The input data can be found in Tables $1(a)$ and $2(a)$, the output data in Table $2(b)$.

optimal play. To find the exact answer to this binary problem one would, however, have to go into details about the exact geometry of the ships and the hydrodynamic effects of close passages.

\section{Numerical experience}

The most advantageous property of the solution algorithm is the astonishing insensitivity of convergence to the initial guesses for the shipmasters' strategies. In all our examples the initial guess is simply that the shipmasters stand on at maximum forward speed. This guess produces optimal solutions after a few iterations in simple cases with few ships involved. 


\begin{tabular}{lrrrrrl}
\hline Ship & 1 & 2 & 3 & 4 & \multicolumn{1}{c}{5} & [units] \\
\hline Initial course & $180 \cdot 0$ & $270 \cdot 0$ & $0 \cdot 0$ & $45 \cdot 0$ & $315 \cdot 0$ & degrees \\
Initial velocity & $14 \cdot 0$ & $11 \cdot 18$ & $14 \cdot 0$ & $11 \cdot 66$ & $14 \cdot 0$ & knots \\
Initial $(x)$ & $500 \cdot 0$ & $0 \cdot 0$ & $-500 \cdot 0$ & $-294 \cdot 0$ & $-350 \cdot 0$ & meters \\
Coordinates $(y)$ & $0 \cdot 0$ & $399 \cdot 0$ & $0 \cdot 0$ & $-294 \cdot 0$ & $350 \cdot 0$ & meters \\
\hline
\end{tabular}

Table 2(a). Initial conditions in fig. (2).

\begin{tabular}{|c|c|c|c|c|c|c|}
\hline \multicolumn{3}{|c|}{$\begin{array}{r}\text { CPA } \\
\text { [metres] }\end{array}$} & \multicolumn{3}{|c|}{ Ships } & \\
\hline $\begin{array}{l}\text { Collision } \\
\text { risk }\end{array}$ & & 1 & 2 & 3 & 4 & 5 \\
\hline & $\begin{array}{l}1 \\
2 \\
3 \\
4 \\
5\end{array}$ & $\begin{array}{l}0.42 \times 10^{-2} \\
0.58 \times 10^{-8} \\
0.40 \times 10^{-4} \\
0.19 \times 10^{-4}\end{array}$ & $\begin{array}{l}258 \\
0 \cdot 64 \times 10^{-3} \\
0 \cdot 28 \times 10^{-3} \\
0 \cdot 20 \times 10^{-2}\end{array}$ & $\begin{array}{l}{ }^{411} 308 \\
0 \cdot 12 \times 10^{-1} \\
0 \cdot 74\end{array}$ & $\begin{array}{l}291 \\
328 \\
178\end{array}$ & $\begin{array}{r}302 \\
278 \\
25 \\
59\end{array}$ \\
\hline
\end{tabular}

Table $2(b)$. Output data for fig. (2).

The CPU-time consumed in one iteration is limited by the relation

$$
\text { CPU } \alpha n(n-1) / 2
$$

where $n$ is the number of ships and installations involved in the problem and $\alpha$ is a constant of proportionality. The CPU-time consumed in one iteration in a problem with $n$ objects is thus approximately proportional to the number of sides and diagonals in a $n$-tagon. The number of iterations that will be needed to attain a solution depends on the stopping criterion. The stopping criterion

$$
\left|\frac{P_{i j} \text { (old) }}{P_{i j} \text { (new) }}-1\right|<0.01 \quad \forall i \in M, \quad \forall j \in M \cup I
$$

will give as a typical result:

number of iterations $=4 \times$ number of ships

A stopping criterion of unchanged strategies at each instant of time for all the players will, however, not always be met with successful termination. This is due to small oscillations near the transition surfaces.

\section{Conclusions}

It has been demonstrated how the analytical theory of differential games formulated with an integral payoff function can be applied to determine optimal evasive manoeuvres for encounters between ships in an environment of other ships and fixed installations. In addition to the numerical examples given in this paper the mathematical model and the solution algorithm could be applied to situations where the shipmasters act as pursuers, or situations where they do not act at all. In fact, the optimal evasive or persuasive manoeuvre can be determined for any predefined strategy of the other ships. 
An obvious criticism of our model is that it is oversimplified. It is well known that the eqns (3) and (4) are coupled due to physical effects like the influence of rudder angle on the acceleration or deceleration, and vice versa; the influence of deceleration on the ship's turning capability. The time lag from rudder command up to onset of actual turn is another important effect that has not been taken care of. These objections do not, however, alter our impression of a realistic mathematical description. Most of the refinement of the dynamic model can also be carried out under the conviction that it will not complicate the solution algorithm. This is especially true for the inclusion of wind and wave forces.

\section{Recommendation for future work}

The only objective of our players has been to minimize the total collision risk. This stems from the formulation of the problem of interest in a particular project. To make the mathematical model more in accordance with the shipmasters' objective, time minimizing or fast recovery from the evasive manoeuvre should be built into the model. This could be done by reformulating the payoff function or by introducing penalties on the use of controls. A mathematical formulation that takes such considerations into account could be applied in ship-based collision avoidance systems and could eventually lead to applications in automatic ship-control systems.

A more fundamental question also remains to be answered. No convergence theorems for the ping-pong algorithm has been given. Our numerical experience indicates that unique solutions are attained in the solution search, which are insensitive to the initial guess on the player's strategies. However, necessary and sufficient conditions for convergence, yet remains to be found.

\section{ACKNOWLEDGMENT}

This study has been financially supported by SPS (Safety Offshore), a research programme under the Royal Norwegian Council for Scientific and Industrial Research.

\section{REFERENCES}

Breakwell, J. V. and Merz, A. W. (1969). Toward a complete solution of the Homicidal Chauffeur Game. Proceedings of the Ist International Conference on the Theory and Application of Differential Games, A. Herst, Mass., pp. HII-1 to III-5.

Holt, D. and Mukundan, R. (1972). A Nash algorithm for a class of non-zero sum differential games. Int. J. Systems Sci., 1972, 2, 4, 379-387.

IsaACs, R. (1965). Differential Games. New York, Krieger Publishing Company.

Jensen, A. (1970). Safety at Sea problems. Accident Analysis and Prevention, I, 1.

Merz, A. W. (1973). Optimal Evasive Manoeuvres in Maritime Collision Avoidance. Navigation, 20, 144-152.

MiLoH, T. (1974). Determination of Critical Manoeuvres for Collision Avoidance Using the Theory of Differential Games. Institut für Shiffbau, Hamburg, Bericht Nr. 319.

Miloh, T. and Sharma, S. D. (1975). Maritime Collision Avoidance as a Differential Game. Institut für Shiffbau, Hamburg, Bericht Nr. 329.

Mjelde, K. (1977). An Analytical Minefield Evaluation Model without Space Averages. Naval res. log. quarterly, 24, 4, 640-650.

SCHroeter, G. (1976). Probability of Kill and Expected Destroyed Value if the Underlaying Distributions are Rotationally Symmetric. Opns. Res., 24, 586-591.

Stratton, A. (1971). Navigation, Traffic and the Community. Presidential Address. The institute of Navigation. The Journal, 24, 1.

Vincent, T. L., Cliff, E. M., Grantham, W. J., and Peng, W. Y. (1972). A problem of Collision Avoidance. University of Arizona, Tucson, EES Series Rep. No. 39. 